\title{
SGLT2-Hemmer als Ursache für die Entstehung einer Ketoazidose
}

\section{Anamnese}

Ein aus Saudi-Arabien stammender 57-jähriger Patient stellte sich in Begleitung eines Dolmetschers erstmals in unserer Diabetesambulanz zur Stoffwechseleinstellung eines vor 13 Jahren diagnostizierten Diabetes mellitus Typ 2 vor. Eindeutige Hinweise auf einen Typ-1-Diabetes lagen angesichts fehlender Ketoazidose bei Erstmanifestation sowie der positiven Familienanamnese für Typ-2-Diabetes nicht vor. Diabetesbedingte Komplikationen wie Nephropathie, Retinopathie oder Polyneuropathie waren nicht bekannt. Sowohl Ketoazidosen als auch Hypoglykämien in der Vergangenheit wurden verneint. Unter oraler glukosesenkender Medikation mit Sitaglipitin (100 mg $1 \times$ täglich) und Glimepirid (4 mg $1 \times$ täglich) war die Blutglukoseeinstellung nicht zufriedenstellend $\left(\mathrm{HbA}_{1 \mathrm{c}}: 85,79 \mathrm{mmol} / \mathrm{mol}\right.$ oder $\left.10 \%\right)$.

Weitere Vorerkrankungen waren bei dem Patienten nicht bekannt. Drogenkonsum wurde verneint. Der Patient gab einen gelegentlichen Alkoholkonsum im Allgemeinen und einen regelmäßigen Alkoholkonsum auf Geschäftsreisen an, ohne genauere Angaben zur Trinkmenge zu machen. In der Familienanamnese fand sich sowohl bei der Mutter als auch beim Vater ein Diabetes mellitus Typ 2.

Die körperliche Untersuchung zeigte einen adipösen (Body Mass Index 31,1 kg/ $\mathrm{m}^{2}$ ) Patienten in einem guten Allgemeinzustand. Ansonsten wurden keine Pathologien erhoben, insbesondere die Auskultation von Herz und Lunge war unauffällig.

Der Patient wünschte eine Therapie unter Vermeidung der Gabe von Insulin. Aus diesem Grund wurde eine orale glukosesenkende Therapie mit dem SGLT2Inhibitor Empagliflozin (10 mg $1 \times$ täglich) begonnen. Die Therapie wurde ergänzt durch das GLP-1-Analagon Dulaglutid, das $1 \times$ wöchentlich in einer Dosis von $0,75 \mathrm{mg}$ subkutan nach vorheriger Schulung des Patienten injiziert werden sollte. Im Rahmen der Schulung wurde der Patient ausführlich über mögliche Nebenwirkungen der neuen Therapie aufgeklärt. In diesem Zusammenhang wurde sonografisch eine Cholezystolithiasis ausgeschlossen und eine Pankreatitis in der Vergangenheit durch den Patienten verneint. Auf eine

- Tab. 1 Venöse Blutgasuntersuchung.

\begin{tabular}{|l|l|l|}
\hline Parameter & Wert & Normbereich \\
\hline $\mathrm{pH}-\mathrm{Wert}$ & 7,184 & $7,35-7,45$ \\
\hline $\mathrm{pCO}_{2}[\mathrm{mmHg}]$ & 19,2 & $35-45$ \\
\hline $\mathrm{PO}_{2}[\mathrm{mmHg}]$ & 47,4 & $71-104$ \\
\hline $\mathrm{HCO}_{3}$ Standard [mmol/l] & 10,3 & $22-27$ \\
\hline $\mathrm{HCO}$ aktuell [mmol/l] & 7,0 & $22-27$ \\
\hline $\mathrm{BE}[\mathrm{mmol} / \mathrm{l}]$ & $-20,4$ & \\
\hline $\mathrm{Sauerstoffsättigung} \mathrm{[ \% ]}$ & 80,9 & $94-99$ \\
\hline Clukose [mg/dl] & 269 & $70-105$ \\
\hline Laktat [mg/dl] & 16 & $4,5-14,4$ \\
\hline BE: Base-Excess. & & \\
\hline
\end{tabular}

leitliniengerechte Therapie mit Metformin wurde angesichts einer anamnestisch berichteten Unverträglichkeit, für die der Patient aber keine genaueren Angaben machen konnte, zunächst verzichtet. Außerdem sollte die orale glukosesenkende Therapie mit Glimepirid nicht fortgeführt werden. 11 Tage später stellte sich der Patient in der zentralen Notaufnahme eines anderen Krankenhauses mit Übelkeit, Erbrechen und diffusen Bauchschmerzen vor. Die Anamnese war aufgrund der Sprachbarriere erschwert. Der Sohn des Patienten gab aber an, sein Vater habe in den vergangenen Tagen vermehrt Alkohol konsumiert, ohne genauere Angaben machen zu können. Fieber als möglicher Hinweis auf einen Infekt bestand nicht und operative Eingriffe in den letzten Tagen wurden durch den Sohn verneint.

\section{Körperliche Untersuchung}

In der zentralen Notaufnahme fiel bei dem Patienten eine Tachypnoe mit einer Atemfrequenz von 24/min auf. Der Patient befand sich in einem noch respiratorisch und hämodynamisch stabilen Zustand. Der Blutdruck war mit 150/100 mmHg erhöht, auch bestand ein tachykarder Sinusrhythmus von 130/min. Die körperliche Untersuchung zeigte einen deliranten und zunehmend somnolenten Patienten ohne Hinweis auf ein fokales neurologisches Defizit, aber mit druckschmerzhaftem Epigastrium.

\section{Diagnostik}

Die Laborbefunde zeigten eine metabolische Azidose ( $>$ Tab. 1). Der Laktatspiegel war geringgradig erhöht. Sowohl ein akutes Nierenversagen als auch eine akute Alkoholintoxikation wurden als Ursache ausgeschlossen. Bei einer Plasmaglukosekonzentration von $269 \mathrm{mg} / \mathrm{dl}$ und dem Nachweis von Ketonen im Urin ( $\triangleright$ Tab. 2) wurde schließlich die Diagnose einer diabetischen Ketoazidose gestellt.

Das Labor ergab außerdem eine Erhöhung des Lipasespiegels im Sinne einer begleitenden Pankreatitis. Eine biliäre Genese ergab sich bei einem unauffälligen Bilirubin nicht. Außerdem zeigte eine Sonografie des Abdomens einen schmalen Ductus hepatocholedochus ohne Hinweis auf Cholestase sowie eine unauffällige Pankreasmorphologie. Der CRP-Wert war mit 3,5 mg/dl nur leicht erhöht ( $\triangleright$ Tab. 3). Eine Röntgenthoraxuntersuchung ergab keinen Hinweis auf das Vorliegen von Infiltraten, und die weitere sonografische Untersuchung des Abdomens zeigte keinen Infektfokus. Eine Leukozyturie als möglicher Hinweis auf eine Harnwegsinfektion war nicht nachweisbar ( $\triangleright$ Tab. 2). 
- Tab. 2 Urinstatus.

\begin{tabular}{|l|l|l|}
\hline Parameter & Wert & Normbereich \\
\hline Spezifisches Gewicht & 1,020 & $1,016-1,022$ \\
\hline $\mathrm{pH}$ im Urin & 6,0 & $4,8-7,4$ \\
\hline Leukozyten & negativ & negativ \\
\hline Nitrit & negativ & negativ \\
\hline Protein & +++ & negativ \\
\hline Glukose & ++++ & negativ \\
\hline Keton & +++++ & negativ \\
\hline Erythrozyten & ++++ & negativ \\
\hline
\end{tabular}

- Tab. 3 Weitere Laboruntersuchungen.

\begin{tabular}{|l|l|l|}
\hline Parameter & Wert & Normbereich \\
\hline Leukozyten [Tsd/Ml] & 11,83 & $4,0-11,0$ \\
\hline C-reaktives Protein [mg/dl] & 3,5 & $0-0,5$ \\
\hline Kreatinin [mg/dl] & 1,2 & $0,5-1,3$ \\
\hline Bilirubin gesamt [mg/dl] & 0,8 & $0,2-1,1$ \\
\hline Lipase [U/l] & 500 & $13-60$ \\
\hline Ethanol Vol [\%] & negativ & negativ \\
\hline
\end{tabular}

\section{Therapie und Verlauf}

Aufgrund der diabetischen Ketoazidose und des zunehmend somnolenten Zustands wurde der Patient zunächst auf die Intensivstation verlegt. Neben einer einmaligen Hämodialyse und bilanzierten Flüssigkeitstherapie erfolgte eine intravenöse Insulintherapie über einen Perfusor entsprechend einem krankenhausinternen Therapiealgorithmus mit etwa 2 I. E. Humaninsulin pro Stunde und einem Blutglukosezielwert von 200-250 mg/dl. Auf die Gabe eines Antibiotikums wurde angesichts fehlenden Fiebers und Infektfokus verzichtet. Die Ketoazidose wurde als potenzielle Nebenwirkung des neu angesetzten SGLT2-Inhibitors Empagliflozin bei gleichzeitigem, durch den Sohn des Patienten berichtetem vermehrten Alkoholgenusses angesehen. Aus diesem Grund wurde die medikamentöse Therapie mit Empagliflozin sofort beendet.

Die begleitende Pankreatitis wurde als mögliche Nebenwirkung der GLP-1-Analogon-Therapie mit Dulaglutid in Verbindung mit dem vermehrten Alkoholgenuss gedeutet. Diese medikamentöse Therapie wurde daher ebenfalls abgesetzt.
Bereits wenige Stunden nach Beginn der Akuttherapie war die metabolische Azidose wieder ausgeglichen, sodass eine weitere Hämodialyse nicht mehr notwendig war. Am Folgetag waren auch im Urin Ketone nicht mehr nachweisbar, sodass die Verlegung auf die Normalstation ohne weitere Optimierung der glukosesenkenden Therapie erfolgte. Nach 3 Tagen wurde der Patient schließlich auf seinen Wunsch hin entlassen und stellte sich 7 Tage nach Entlassung aus dem Krankenhaus wieder in unserer Diabetesambulanz vor.

Angesichts der metabolischen Azidose und begleitenden Pankreatitis wurde die Medikation mit Empagliflozin und Dulaglutid nicht wieder begonnen. Auf eine Wiederaufnahme der Therapie mit einem DPP4-Hemmer wurde angesichts der kürzlich aufgetretenen Pankreatitis ebenfalls bewusst verzichtet. Außerdem sprach sich der Patient erneut gegen eine Insulintherapie aus. Daher leiteten wir auf Wunsch des Patienten und bei inzwischen normalisiertem Laktatspiegel eine Therapie mit Metformin ein mit dem Hinweis, dass Metformin allein nicht zu einer adäquaten Stoffwechselein- stellung führen wird. Bei jedoch nicht sicher auszuschließender Unverträglichkeit begannen wir mit einem niedrigdosierten Titrationsschema (initial $500 \mathrm{mg}$ einmal täglich, Steigerung nach 3 Tagen bei guter Verträglichkeit auf zweimal täglich).

\section{Diskussion}

Die SGLT2-Inhibitoren haben sich in der Therapie des Diabetes mellitus Typ 2 seit Jahren bewährt und etabliert, vor allem bei Patienten mit kardiovaskulären Erkrankungen [1] und diabetischer Nephropathie [2]. In Deutschland sind Dapagliflozin und Empagliflozin zugelassen. Seit Anfang des Jahres besteht auch für die Therapie des Diabetes mellitus Typ 1 die Zulassung für Dapagliflozin in einer Dosis von $5 \mathrm{mg} 1 \times$ täglich.

Jedoch geht die Anwendung von SGLT2-Inhibitoren mit einer Reihe von Nebenwirkungen einher [3]. So erhöhen sie etwa, wenn auch selten, das Risiko für eine diabetische Ketoazidose, eine potenziell lebensbedrohliche akute Komplikation des Diabetes [4]. Die US-amerikanische Arzneimittelbehörde Food and Drug Administration (FDA) warnte 2015 in einer Drug Safety Communication erstmals vor der Gefahr einer diabetischen Ketoazidose unter der Einnahme von SGLT2-Inhibitoren [5]. In der Folge durchgeführte Metaanalysen kontrollierter klinischer Studien ergaben Häufigkeiten von durch SGLT2-Inhibitor induzierten diabetischen Ketoazidosen von weniger als $0,1 \%$, ohne dass jedoch ein erhöhtes Risiko gegenüber den Vergleichsgruppen nachzuweisen war [6, 7]. Hingegen war in einer Auswertung von Daten US-amerikanischer Krankenversicherten das Risiko für mit SGLT2-Inhibitor assoziierte diabetische Ketoazidosen nahezu verdoppelt im Vergleich zu einer Therapie mit DPP4-Inhibitoren [8]. Eine aktuelle Metaanalyse randomisierter kontrollierter Studien zeigte wiederum kein erhöhtes Risiko für eine diabetische Ketoazidose unter der Einnahme von SGLT2Inhibitoren im Vergleich zu Plazebo [9]. Allerdings konnten aufgrund der weiten Konfidenzintervalle, als Ausdruck einer eingeschränkten Genauigkeit, klinisch wichtige Nebenwirkungen nicht ausgeschlossen werden.

Wie können SGLT2-Inhibitoren das Auftreten einer Ketoazidose fördern? Es wer- 
den verschiedene Mechanismen diskutiert. SGLT2-Hemmer führen zum einen zu einer verminderten Rückresorption von Glukose aus dem Primärharn in den proximalen Nierentubuli und damit zur vermehrten Glukoseausscheidung über den Harn und zur Senkung des Blutglukosespiegels. Gerade dieser blutglukosesenkende Effekt hat den Abfall des Insulinspiegels zur Folge aufgrund einer verminderten Insulinausschüttung aus den Beta-Zellen der Bauchspeicheldrüse. Gegenregulatorisch wird die Freisetzung von Glukagon aus den AlphaZellen der Bauchspeicheldrüse gesteigert. Sowohl der Anstieg von Glukagon im Blut als auch die vermehrte Lipolyse und Fettsäureoxidation infolge der verminderten Insulinausschüttung führen schließlich zur vermehrten Ketogenese in der Leber [10, 11]. In der Folge kommt es zum Anstieg von Ketonkörpern im Blut und zum Auftreten einer Ketoazidose ( $\triangleright$ Abb. 1). Darüber hinaus wird vermutet, dass Alpha-Zellen im Pankreas SGLT2-Transporter exprimieren, die als Glukosesensoren dienen [12]. Kommt es zu einer Blockade der Transporter durch SGLT2-Hemmer, wird eine Hypoglykämie vorgetäuscht und es kommt ebenfalls zu einer vermehrten Ausschüttung von Glukagon. Infolge der durch SGLT2-Hemmer vermittelten verminderten Natriumrückresorption aus dem Primärharn in den proximalen Nierentubuli kommt es zum Anstieg des elektrochemischen Gradienten und so zu einer gesteigerten Rückresorption von Ketonkörpern, insbesondere Acetoacetat, in der Niere. Daraus resultiert schließlich ein weiterer Anstieg der Ketonkörper im Blut [10]. Aus diesem Grund kann sich unter einer Therapie mit einem SGLT2-Hemmer eine Ketoazidose entwickeln, ohne dass Ketonkörper im Urin nachzuweisen sind [13].

Typische Diagnosekriterien sind eine hyperglykämische Dekompensation (Blutglukosewert > $250 \mathrm{mg} / \mathrm{dl}$ bzw. > $14 \mathrm{mmol} /$ I), Nachweis einer Ketonurie und eine metabolische Azidose ( $\mathrm{pH}$-Wert <7,3, Serumbikarbonat $\leq 15 \mathrm{mmol} / \mathrm{l}$ und Anionengap > 12 mmol// [11]. Diese Laborkonstellation zeigte sich auch bei unserem Patienten. Darüber hinaus gab es auch Fälle mit Ketoazidosen ohne klinisch relevante hyperglykämische Dekompensation. In diesen Fällen liegt eine euglykämische Ketoazidose vor [13, 14]. Eine durch SGLT2-Hemmer induzierte euglykämische

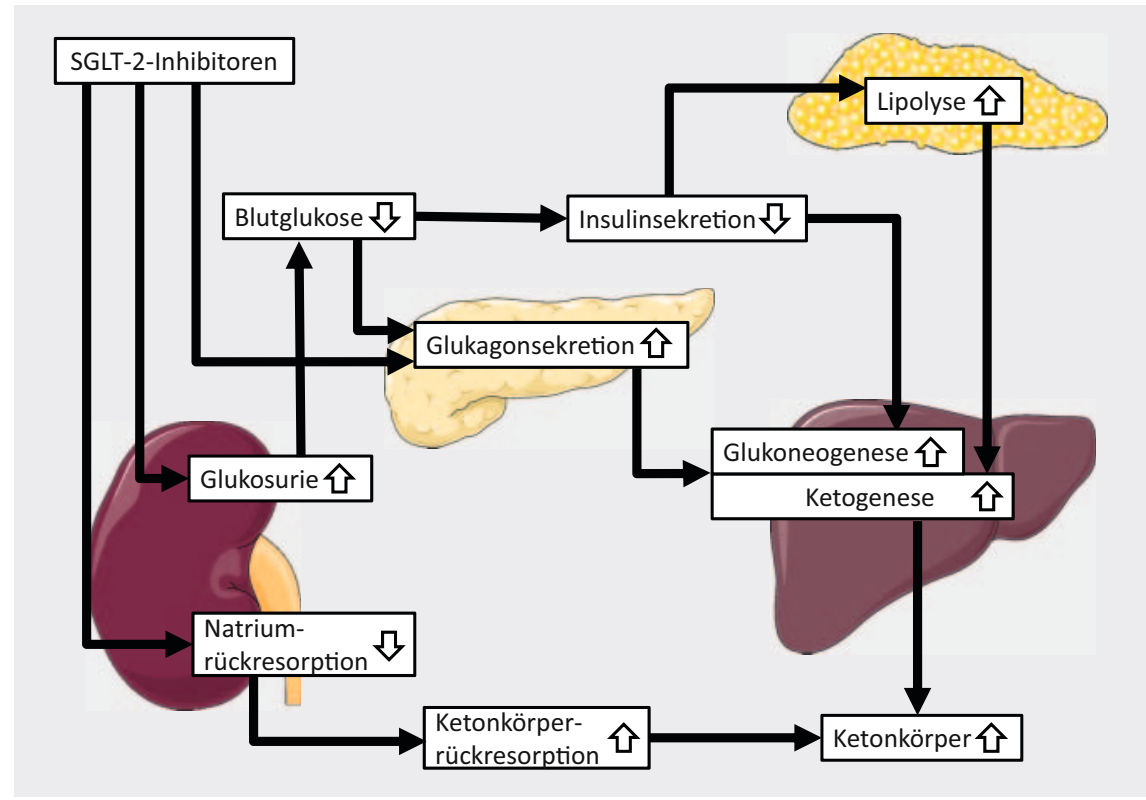

- Abb.1 Mechanismen der durch SGLT2-Inhibitor induzierten Ketoazidose.

diabetische Ketoazidose scheint durch Dehydratation und Insulinmangel getriggert zu werden [15]. Durch das zusätzliche Fehlen von Ketonkörpern im Urin kann so die Diagnose einer diabetischen Ketoazidose erschwert werden. Dies kann zu einer verzögerten Diagnosestellung und schließlich zu lebensbedrohlichen Komplikationen wie etwa Volumenmangelschock und Koma bis hin zum Tod des Patienten führen.

Aus diesem Grund ist es umso wichtiger, alle Patienten, die mit einem SGLT2-Inhibitor behandelt werden, auf die Symptome einer diabetischen Ketoazidose hinzuweisen (Box 1), über die auch unser Patient zum Teil klagte. Beim Auftreten dieser Symptome sollte unabhängig vom Blutglukosespiegel eine Ketoazidose in Betracht gezogen und die Behandlung mit einem SGLT2Inhibitor unverzüglich beendet werden.

\section{BOX 1}

Symptome der diabetischen Ketoazidose

- Polyurie, Polydipsie

- Übelkeit, Erbrechen

- Diffuse Bauchscherzen

- Müdigkeit/Erschöpfung

- Verwirrtheit/Delir

- Kussmaul-Atmung

- Azetongeruch
Auch gibt es Faktoren, die das Auftreten einer Ketoazidose unter einer Therapie mit einem SGLT2-Hemmer begünstigen (Box 2), über die der Patient aufgeklärt werden muss. Vor Beginn einer Behandlung sind diese Faktoren in der Anamnese des Patienten zu eruieren. Unter diesen Umständen sollte die Einleitung der Therapie mit einem SGLT2-Hemmer genau abgewogen oder eine bereits bestehende Therapie pausiert werden. In unserem Fall hat möglicherweise der vom Sohn berichtete vermehrte Alkoholgenuss das Auftreten der Ketoazidose unter Empagliflozin begünstigt.

\section{BOX 2}

Begünstigende Faktoren einer diabetischen Ketoazidose

- Akute Infektionen

- Fasten/kohlenhydratarme Diät

- Reduktion der Insulindosis

- Alkohol- und Drogenkonsum

- Operationen

- Körperliche Betätigung

- Dehydratation

Zusammenfassend lässt sich festhalten, dass SGLT2-Inhibitoren bei Patienten mit erhöhtem Risiko für das Auftreten einer diabetischen Ketoazidose mit Vorsicht oder gar nicht angewendet werden soll- 
ten. Dazu gehören Patienten mit Typ-1Diabetes, Patienten mit einem insulinbehandelten Typ-2-Diabetes oder Patienten mit Diabetes infolge einer Erkrankung der exokrinen Pankreasfunktion.

Interessenkonflikt

Die Autoren geben an, dass kein Interessenkonflikt besteht.

\section{Autorinnen/Autoren}

Markus Emmelheinz', Michael Roden 1, 2, 3, Karsten Müssig 1, 2, 3, 4

${ }^{1}$ Klinik für Endokrinologie und Diabetologie, Medizinische Fakultät, Heinrich-HeineUniversität Düsseldorf

${ }^{2}$ Institut für klinische Diabetologie, Deutsches Diabetes-Zentrum, Leibniz-Zentrum für Diabetesforschung an der Heinrich-HeineUniversität Düsseldorf

${ }^{3}$ Deutsches Zentrum für Diabetesforschung (DZD), München-Neuherberg

${ }^{4}$ Klinik für Innere Medizin, Niels-Stensen-Kliniken, Franziskus-Hospital Harderberg, Georgsmarienhütte

Korrespondenzadresse

Prof. Dr. med. Karsten Müssig

Klinik für Innere Medizin

Niels-Stensen-Kliniken

Franziskus-Hospital Harderberg

Alte Rothenfelder Str. 23

49124 Georgsmarienhütte

Karsten.Muessig@niels-stensen-kliniken.de
Literatur

[1] Zinman B, Wanner C, Lachin JM et al. EMPAREG OUTCOME Investigators. Empagliflozin, Cardiovascular Outcomes, and Mortality in Type 2 Diabetes. N Engl J Med 2015; 373: 2117-2128

[2] Wiviott SD, Raz I, Bonaca MP et al. DECLARETIMI 58 Investigators. Dapagliflozin and Cardiovascular Outcomes in Type 2 Diabetes. N Engl J Med 2019; 380: 347-357

[3] Beitelshees AL, Leslie BR, Taylor SI. SodiumGlucose Cotransports 2 inhibitors: A Case Study in Translational Research. Diabetes 2019; 68: 1109-1120

[4] Hamblin PS, Wong R, Ekinci El et al. SGLT2 Inhibitors Increase the Risk of Diabetic Ketoacidosis Developing in the Community and During Hospital Admission. J Clin Endocrinol Metab 2019; 104: 3077-3087

[5] U.S. Food and Drug Administration. FDA warns that SGLT2 inhibitors for diabetes may result in a serious condition of too much acid in the blood: U.S. Food and Drug Administration. 2015

[6] Tang H, Li D, Wang T et al. Effect of SodiumGlucose Cotransporter 2 Inhibitors on Diabetic Ketoacidosis Among Patients With Type 2 Diabetes: A Meta-analysis of Randomized Controlled Trials. Diabetes Care 2016; 39: e123-e124

[7] Monami M, Nreu B, Zannoni S et al. Effects of SGLT-2 inhibitors on diabetic ketoacidosis: A meta-analysis of randomised controlled trials. Diabetes Res Clin Pract 2017; 130: 53-60

[8] Fralick M, Schneeweiss S, Patorno E. Risk of Diabetic Ketoacidosis after Initiation of an SGLT2 Inhibitor. N Engl J Med 2017; 376: 2300-2302
[9] Donnan JR, Grandy CA, Chibrikov E et al. Comparative safety of the sodium glucose co-transporter 2 (SGLT2) inhibitors: a systematic review and meta-analysis. BMJ Open 2019: 9

[10] Taylor SI, Blau JE, Rother KI. SGLT2 Inhibitors May Predispose to Ketoacidosis. J Clin Endocrinol Metab 2015; 100: 2849-2852

[11] Goldenberg RM, Berard LD, Cheng AYY et al. SGLT2 Inhibitor-associated Diabetic Ketoacidosis: Clinical Review and Recommendations for Prevention and Diagnosis. Clin Ther 2016; 38: 2654-2664

[12] Bonner C, Kerr-Conte J, Gmyr V et al. Inhibition of the glucose transporter SGLT2 with dapagliflozin in pancreatic alpha cells triggers glucagon secretion. Nat Med 2015; 21 : 512-517

[13] Milder DA, Milder TY, Kam PCA. Sodiumglucose co-transporter type-2 inhibitors: pharmacology and peri-operative considerations. Anaesthesia 2018; 73: 1008-1018

[14] Rosenstock J, Ferrannini E. Euglycemic diabetic ketoacidosis: a predictable, detectable, and preventable safety concern with SGLT2 inhibitors. Diabetes Care 2015; 38: 16381642

[15] Perry RJ, Rabin-Court A, Song JD et al. Dehydration and insulinopenia are necessary and sufficient for euglycemic ketoacidosis in SGLT2 inhibitor-treated rats. Nat Commun 2019; 10: 548

Bibliografie

DOI https://doi.org/10.1055/a-1011-8694

Diabetologie 2019; 14: 439-442

(c) Georg Thieme Verlag KG, Stuttgart · New York ISSN 1861-9002 